\title{
Proton-Induced Charge Transfer on Imidazole and 2- Aminoimidazole. Role of the Substituent and Influence of Stepwise Hydration
}

Boutheïna Kerkeni ${ }^{1,2,3}$ and Marie-Christine Bacchus-Montabonel $\mathrm{l}^{3^{*}}$

1 Département de Physique, laboratoire de Physique de la Matière Condensée, Faculté des Sciences de Tunis, Université de Tunis el Manar, Campus Universitaire 2092 Tunis, Tunisia 2 Université de la Manouba, Institut Supérieur des Arts Multimédia de la Manouba, 2010 la Manouba, Tunisia

3 Univ Lyon, Université Claude Bernard Lyon 1, CNRS, Institut Lumière Matière, F-69622, Villeurbanne, France

*bacchus@univ-lyon1.fr

Table S1 Structures of the imidazole@ $\left(\mathrm{H}_{2} \mathrm{O}\right)_{\mathrm{n}}$ clusters

$\begin{array}{lrrr}\mathrm{n}=1 & & \\ \mathrm{C} & 0.630803000 & -0.974885000 & 0.000000000 \\ \mathrm{~N} & -0.741162000 & -0.964252000 & 0.000000000 \\ \mathrm{C} & -1.075551000 & 0.279377000 & 0.000000000 \\ \mathrm{~N} & 0.000000000 & 1.095109000 & 0.000000000 \\ \mathrm{C} & 1.110055000 & 0.287933000 & 0.000000000 \\ \mathrm{H} & -2.078690000 & 0.655404000 & 0.000000000 \\ \mathrm{H} & -0.013611000 & 2.086916000 & 0.000000000 \\ \mathrm{H} & 2.104819000 & 0.677707000 & 0.000000000 \\ \mathrm{H} & 1.183777000 & -1.890569000 & 0.000000000 \\ \mathrm{O} & 2.221147000 & 0.621254000 & 2.050285000 \\ \mathrm{H} & 2.832102000 & 1.316835000 & 2.368323000 \\ \mathrm{H} & 2.774972000 & 0.147270000 & 1.386545000\end{array}$

$\begin{array}{lrrr}\mathrm{n}=2 & & & \\ \mathrm{C} & 0.743781694 & -1.234140010 & -0.099855269 \\ \mathrm{~N} & -0.646732207 & -1.313232756 & -0.124744567 \\ \mathrm{C} & -1.071839262 & -0.045851646 & -0.112822538 \\ \mathrm{~N} & -0.025208344 & 0.841417732 & -0.082000885 \\ \mathrm{C} & 1.146749077 & 0.088280188 & -0.070056129 \\ \mathrm{H} & -2.103594134 & 0.263401600 & -0.116341381 \\ \mathrm{H} & -0.065468337 & 1.870983909 & 0.006704443 \\ \mathrm{H} & 2.120607505 & 0.548375621 & -0.105968313 \\ \mathrm{H} & 1.352425096 & -2.123351811 & -0.115015649 \\ \mathrm{O} & 1.332791558 & 1.910976466 & 2.619634193 \\ \mathrm{H} & 2.134385732 & 1.893531430 & 3.169282472 \\ \mathrm{H} & 1.169778854 & 1.036482390 & 2.211896677 \\ \mathrm{O} & 0.256252148 & 3.414613339 & 0.741842905 \\ \mathrm{H} & 0.707545829 & 3.077435023 & 1.563470927 \\ \mathrm{H} & -0.337663561 & 4.159148220 & 0.930275432\end{array}$


1.255235840

0.188625742

$-1.018255293$

$-0.663141208$

0.699127298

1.854742368

1.189464990

$-1.347263695$

1.204599908

2. 320568196

0.204782430

1.706443645

0.689836619

1.656174702

1.844043839

$-0.013138048$

$-0.976754476$

0.411583650
$-0.777077408$

$-1.662335926$

$-0.955438411$

0.335012812

0.491808514

2.626027715

1. 738778946

1.166709924

1.386701101

$-0.935540696$

$-2.738823229$

1. 766958879

0.870915436

2.385648953

3.583049658

$-0.571816887$

$-0.599550435$

$-1.044229182$
$-0.183188850$

$-0.208423423$

$-0.204025283$

$-0.180984514$

$-0.171506208$

0.880113573

3.209769874

$-0.160671840$

0.020071230

$-0.194054933$

$-0.258665339$

4.030181740

3.120079733

1.844428531

0.723265598

2.821041124

2.679204558

2.071989866

\section{$\mathrm{n}=4$}

$\mathrm{N}$

C

C

$\mathrm{N}$

C

0

0

O

$\mathrm{H}$

$\mathrm{H}$

$\mathrm{H}$

$\mathrm{H}$

$\mathrm{H}$

$\mathrm{H}$

$\mathrm{H}$

$\mathrm{H}$

$\mathrm{H}$

$\mathrm{H}$

O

$\mathrm{H}$

$\mathrm{H}$
$-0.047459000$

1. 310480000

1.956477000

1.007660000

$-0.186778000$

$-2.527764000$

$-3.320998000$

$-1.926899000$

$-1.144281000$

$-0.882003000$

1.691814000

3.012561000

$-4.044903000$

$-2.774379000$

$-2.888174000$

$-2.914989000$

$-1.093638000$

$-1.852122000$

0.219103000

0.305650000

0.737482000
1.096821468

1. 377912752

0.160392437

$-0.858438643$

$-0.251858979$

1. 901247763

$-0.514089499$

$-2.377706768$

$-0.734128466$

1.712044220

2. 381895826

$-0.049692802$

$-0.911996344$

$-1.237145940$

0.988686933

2.598676979

$-2.755964554$

$-2.452832102$

$-3.161492543$

$-3.949241866$

$-2.349318920$
0.300117484

0.257750218

0.328923245

0.415631136

0.391270636

0.800549421

1.387004961

2.451677611

0.463389093

0.376630091

0.186045355

0.326486406

0.876322696

1.865623063

1.053092416

1.353498501

1.988602384

3.416195755

1.304886930

0.744978913

0.931188877

\section{$\mathrm{n}=5$}

$\mathrm{C}$

$\mathrm{N}$

C

C

$\mathrm{N}$

O

0

0

O

$\mathrm{H}$

$\mathrm{H}$

H
$-0.011284266$

$-0.287191442$

0.919529724

1. 908497391

1. 315077243

$-0.283173316$

$-2.376115557$

$-3.608798361$

$-2.861444603$

$-0.767701567$

$-1.266345645$

0.971889139
$-0.503529952$

0.828606611

1. 511081288

0.550531125

$-0.709554873$

$-4.061407042$

$-2.929798118$

$-0.837324241$

1. 359809462

$-1.272557532$

1.207710482

2.586184363
1.158524004

1.162755526

1.179737846

1.187909930

1.174757763

$-0.153291363$

0.651066637

$-0.139895359$

0.921037065

1.129036084

1.112073427

1.181248305 


$\begin{array}{rr}\mathrm{H} & 2.978438167 \\ \mathrm{H} & -3.941114417 \\ \mathrm{H} & -3.127109738 \\ \mathrm{H} & -3.223996850 \\ \mathrm{H} & -3.481246883 \\ \mathrm{H} & -1.535954901 \\ \mathrm{H} & -2.714980811 \\ \mathrm{H} & 0.616143580 \\ \mathrm{H} & -0.168094405 \\ \mathrm{O} & 1.839245441 \\ \mathrm{H} & 2.311463724 \\ \mathrm{H} & 1.766911326\end{array}$

$$
\begin{array}{r}
0.672055322 \\
-0.903987394 \\
-1.687810025 \\
0.520010914 \\
1.705107586 \\
-3.404562949 \\
-3.388224562 \\
-3.773581643 \\
-4.388226316 \\
-3.202904024 \\
-3.650559531 \\
-2.162169237
\end{array}
$$

1.199409141 $-1.048271514$

0.153868090

0.465458546

1.582925009

0.269712282

1.436576576

0.275582145

$-1.058606441$

0.878036614

1.597571190

1.017117101

$\begin{array}{lrrr}\mathrm{n}=6 & & & \\ \mathrm{C} & 1.880843829 & -0.656191339 & -1.032192891 \\ \mathrm{~N} & 1.276529238 & 0.595839643 & -1.119402198 \\ \mathrm{C} & -0.048978656 & 0.381645297 & -1.187131427 \\ \mathrm{~N} & -0.312121987 & -0.949144231 & -1.146012418 \\ \mathrm{C} & 0.896845542 & -1.621207865 & -1.046965307 \\ \mathrm{O} & 1.817348308 & 3.010270259 & -0.740302337 \\ \mathrm{O} & -0.206577535 & 3.764866102 & 0.425597784 \\ \mathrm{O} & -2.202083577 & 3.002374027 & -1.087394144 \\ \mathrm{O} & -3.079284220 & 0.920394796 & 0.327133361 \\ \mathrm{O} & -2.833810838 & -1.353717909 & -0.743374477 \\ \mathrm{O} & -1.015320769 & 1.630440839 & 1.861189653 \\ \mathrm{H} & -0.808189361 & 1.145858154 & -1.249293819 \\ \mathrm{H} & -1.301205518 & -1.322620411 & -1.086956500 \\ \mathrm{H} & 0.954620249 & -2.694511900 & -0.992220502 \\ \mathrm{H} & 2.949738635 & -0.767302348 & -0.964739796 \\ \mathrm{H} & -2.424819850 & 1.078178167 & 1.065706849 \\ \mathrm{H} & -2.998117082 & 1.693006423 & -0.292460133 \\ \mathrm{H} & -3.048055052 & -0.454515020 & -0.275804518 \\ \mathrm{H} & -3.614384092 & -1.811925214 & -1.087384653 \\ \mathrm{H} & -1.478683829 & 3.420803160 & -0.523099644 \\ \mathrm{H} & -2.419994740 & 3.534787962 & -1.869224546 \\ \mathrm{H} & 0.718449517 & 3.523188632 & -0.071008321 \\ \mathrm{H} & -0.164892394 & 4.580140502 & 0.953765313 \\ \mathrm{H} & 2.297147100 & 3.512454608 & -1.416557954 \\ \mathrm{H} & 1.715018212 & 1.938172384 & -0.950214334 \\ \mathrm{H} & -0.297985907 & 0.992585421 & 2.015117723 \\ \mathrm{H} & -0.667687559 & 2.446528067 & 1.398110501\end{array}$

\section{Table S2 Structures of the 2-aminoimidazole@ $\left(\mathrm{H}_{2} \mathrm{O}\right)_{n}$ clusters}

$\begin{array}{lr}\mathrm{n}=1 & \\ \mathrm{~N} & 0.622738108 \\ \mathrm{C} & 1.245915870 \\ \mathrm{C} & 0.228851180 \\ \mathrm{~N} & -1.012756984 \\ \mathrm{C} & -0.732643109 \\ \mathrm{~N} & -1.655403866 \\ \mathrm{H} & 1.016336187 \\ \mathrm{H} & 2.316907364 \\ \mathrm{H} & 0.303466598\end{array}$

0.583132858
-0.654144506
-1.587972667
-0.950680562
0.351346913
1.431116404
1.525488767
-0.760156667
-2.662567782

$-0.228718281$

$-0.192518807$

$-0.208746306$

$-0.241535909$

$-0.254493052$

$-0.303432071$

$-0.305580029$

$-0.159691790$

$-0.194470673$ 


$\begin{array}{rr}\mathrm{H} & -2.008059377 \\ \mathrm{H} & -2.429979785 \\ \mathrm{O} & 0.412850543 \\ \mathrm{H} & -0.499027369 \\ \mathrm{H} & 0.578494242\end{array}$

\section{$\mathrm{n}=2$}

$\mathrm{N}$

C

C

$\mathrm{N}$

C

$\mathrm{N}$

O

$\mathrm{H}$

$\mathrm{H}$

$\mathrm{H}$

$\mathrm{H}$

$\mathrm{H}$

$\mathrm{H}$

$\mathrm{H}$

O

$\mathrm{H}$

$\mathrm{H}$

\section{$\mathrm{n}=3$}

$\mathrm{C}$

$\mathrm{N}$

C

C

$\mathrm{N}$

$\mathrm{N}$

0

O

$\mathrm{H}$

$\mathrm{H}$

$\mathrm{H}$

$\mathrm{H}$

$\mathrm{H}$

$\mathrm{H}$

$\mathrm{H}$

$\mathrm{H}$

$\mathrm{H}$

O

$\mathrm{H}$

$\mathrm{H}$

\section{$\mathrm{n}=4$}

C

C

$\mathrm{N}$

C

$\mathrm{N}$

$\mathrm{N}$
-0.675962834
0.670230901
1.321587479
0.331992125
-0.925959425
-1.663012524
1.636105797
-0.595610043
1.117213074
2.394096010
0.429721282
-1.742180195
-2.562192705
0.707935190
2.160236075
-1.146294601
-0.184721376
1.384635723
1.536871594
1.844005987

$-0.825587103$

0.484780657

1. 317039618

0.502833215

$-0.802757756$

0.892888968
1.713422735

1.242337447

3.256964807

2. 848261815

3.819114853

0.489962926

$-0.787070902$

$-1.650729271$

$-0.939260128$

0.332750412

1.427459528

2. 992906038

1.399963673

$-0.955219533$

$-2.722006982$

1.942140086

1.103198643

3.348786627

3.677938572

3.434771545

2. 676106546

3.444359209
0.610250646

$-0.933955887$

$-0.657859474$

$-0.687384520$

$-1.434063140$

$-0.054198709$

$-0.183913297$

$-0.280309654$

$-0.199641743$

$-0.069780784$

$-0.001449571$

$-0.300922530$

$-0.049198004$

$-0.196124445$

$-0.393450000$

0.878258274

$-0.192238271$

$-0.866927385$

0.237396882

$-1.546658975$

$-1.094986598$

$-2.508307632$

0.295101260

0.568741502

$-0.663729024$

$-1.620362295$

$-1.012034434$

1. 302425614

3. 056302302

3. 245121798

1.492399328

$-0.738492928$

$-2.692723030$

1. 917396593

0.892849923

3. 361181120

3.768566169

2. 550381154

2. 878675930

2. 157128128

1.223323283

2. 394398989
$-0.296897189$

$-0.251906225$

$-0.189664612$

$-0.214097435$

$-0.272623200$

$-0.407179518$

$-0.980005669$

$-2.121126677$

$-0.359352838$

$-0.127665676$

$-0.183149089$

0.400803931

$-0.644139990$

$-1.332124939$

$-0.577350432$

$-1.658955206$

$-2.948166696$

$-3.544420553$

$-3.770071661$

$-2.699468832$
-1.360193281
-1.745841361
-0.633079595
0.417364009
0.033584265
1.739597662
0.309680042

0.314268742

0.327522074

0.329842022

0.304640622

0.302512691 
3.304625043

1. 747512262

$-0.543636975$

$-1.740524027$

$-1.571178557$

$-1.750168330$

0.892141056

0.199304426

1.827019756

$-1.392416398$

$-2.432530421$

$-0.364787094$

0.282904354

2. 361418310

1.550736136

2. 748761235

4. 218273150
0.349038599

0.620548606

2. 124785051

2.475034890

0.673069433

$-1.916850310$

$-2.747213387$

2.384417550

1. 888740166

2. 377843952

3. 151148061

2. 859742291

1.575097393

0.544693330

$-0.290510684$

$-0.187695370$

0.035281955
1.880739329

4.137756075

4.001806485

1.550180506

0.487184492

0.291620357

0.298300602

0.673839896

0.669755671

2.473446699

1.584290781

4.606158071

3.999976657

3.364289496

4.401310870

1.237834946

1.820166285

\section{$\mathrm{n}=5$}

\section{$\mathrm{C}$}

$\mathrm{N}$

C

$\mathrm{N}$

C

$\mathrm{N}$

0

0

O

0

O

$\mathrm{H}$

$\mathrm{H}$

$\mathrm{H}$

$\mathrm{H}$

$\mathrm{H}$

$\mathrm{H}$

$\mathrm{H}$

$\mathrm{H}$

$\mathrm{H}$

$\mathrm{H}$

$\mathrm{H}$

$\mathrm{H}$

$\mathrm{H}$

$\mathrm{H}$

$\mathrm{H}$
-0.575260279
0.761772885
0.777560981
-0.496449924
-1.372607787
1.876087320
2.832391395
4.681227216
2.289859268
2.465408390
5.246947952
-0.734819950
-2.444265222
-0.870423591
1.984799875
2.741071369
3.443109514
2.275549119
2.494064894
3.021581524
2.033342568
2.830641099
5.160616667
5.795581814
4.151842478
5.290590697

$-1.538640000$

$-1.167766000$

0.163730000

0.648730000

$-0.429827000$

1.000053000

$-2.204505000$

$-0.495986000$

$-0.690875000$

1. 973617000

1.509337000

1.629470000

$-0.301831000$

$-2.578496000$

1.544693000

0.491278000

1. 979764000

1.129748000

$-1.274175000$

$-0.804019000$

$-1.926042000$

$-3.172904000$

0.817929000

2. 210276000

$-1.233716000$

$-0.897628000$
0.384291319

0.418387482

0.432568997

0.417469804

0.393390266

0.423139540

1.789639717

0.711098732

4.161158630

3.093656976

2.610963586

0.372185447

0.391027532

0.367423804

1.293966319

0.250283350

3.017154055

3.557091071

3.397541467

4.785726141

1.217993328

1.826014480

1.916972191

2.229879443

1.104958733

0.074553846

\section{$\mathrm{n}=6$}

$\mathrm{C}$

$\mathrm{N}$

C

$\mathrm{N}$

C

$\mathrm{N}$

O

0

o
1.549342975

1.272227676

$-0.062962114$

$-0.637885360$

0.372646504

$-0.748473924$

2.498704791

0.712316971

1.510833211
0.600887657

$-0.765530410$

$-0.916029032$

0.310078809

1. 274728469

$-2.081571686$

$-2.560938833$

$-4.350686124$

$-1.155100938$
$-0.505374849$

$-0.511331448$

$-0.521248349$

$-0.471269893$

$-0.484222738$

$-0.516830187$

$-1.846186384$

$-1.653603970$

$-3.923364802$ 


$\begin{array}{lrrr}\mathrm{O} & -1.274655142 & -1.471217827 & -3.954919660 \\ \mathrm{O} & -2.837484474 & -0.580379429 & -1.970360918 \\ \mathrm{O} & -0.926978208 & -4.265949503 & -3.875518007 \\ \mathrm{H} & -1.616380625 & 0.437556136 & -0.730096630 \\ \mathrm{H} & 0.151146516 & 2.329976121 & -0.465341282 \\ \mathrm{H} & 2.564629786 & 0.965326591 & -0.518739369 \\ \mathrm{H} & -1.704235868 & -2.005503556 & -0.857509575 \\ \mathrm{H} & -0.249703942 & -2.926052628 & -0.817961241 \\ \mathrm{H} & -2.292047234 & -0.855465304 & -2.769436400 \\ \mathrm{H} & -3.743882898 & -0.451389891 & -2.284726768 \\ \mathrm{H} & -1.275649643 & -2.454557714 & -3.988625870 \\ \mathrm{H} & -0.314320928 & -1.237633601 & -3.939268651 \\ \mathrm{H} & 1.961864799 & -1.704421969 & -3.214899479 \\ \mathrm{H} & 1.832259992 & -1.497378237 & -4.770306122 \\ \mathrm{H} & 1.938085408 & -1.546300999 & -0.930381852 \\ \mathrm{H} & 3.444322276 & -2.756718340 & -1.779391733 \\ \mathrm{H} & -0.317198425 & -4.406852070 & -3.109350688 \\ \mathrm{H} & -1.623432824 & -4.933556068 & -3.799292042 \\ \mathrm{H} & 1.544334808 & -3.733517288 & -1.761294252 \\ \mathrm{H} & 1.000997793 & -5.143670745 & -1.178806657\end{array}$

Table S3 Charge transfer cross sections with regard to impact energy in proton-induced collisions with imidazole and IMI@ $\left(\mathrm{H}_{2} \mathrm{O}\right) \mathrm{n}$ water clusters (in 10-16 $\left.\mathrm{cm}^{2}\right)$.

\begin{tabular}{|l|l|l|l|l|l|l|l|}
\hline $\mathrm{E}_{\text {lab }}(\mathrm{eV})$ & imidazole & IMI@, $1 \mathrm{~W}$ & IMI@2W & IMI@,3W & IMI@,4W & IMI@, $5 \mathrm{~W}$ & IMI@6W \\
\hline 2.5 & $7.73 \times 10^{-4}$ & $9.44 \times 10^{-4}$ & $2.39 \times 10^{-4}$ & $1.68 \times 10^{-4}$ & $2.77 \times 10^{-3}$ & $3.71 \times 10^{-4}$ & $1.05 \times 10^{-4}$ \\
\hline 5.7 & $1.85 \times 10^{-3}$ & $1.63 \times 10^{-3}$ & $2.24 \times 10^{-4}$ & $1.44 \times 10^{-4}$ & $3.49 \times 10^{-3}$ & $5.11 \times 10^{-4}$ & $1.98 \times 10^{-4}$ \\
\hline 10.0 & $2.49 \times 10^{-3}$ & $2.28 \times 10^{-3}$ & $2.20 \times 10^{-4}$ & $1.33 \times 10^{-4}$ & $3.76 \times 10^{-3}$ & $6.73 \times 10^{-4}$ & $2.26 \times 10^{-4}$ \\
\hline 22.6 & $4.78 \times 10^{-3}$ & $3.57 \times 10^{-3}$ & $1.96 \times 10^{-4}$ & $2.55 \times 10^{-4}$ & $3.98 \times 10^{-3}$ & $7.08 \times 10^{-4}$ & $1.02 \times 10^{-3}$ \\
\hline 40.0 & $5.61 \times 10^{-3}$ & $4.78 \times 10^{-3}$ & $3.01 \times 10^{-4}$ & $3.45 \times 10^{-4}$ & $3.71 \times 10^{-3}$ & $7.20 \times 10^{-4}$ & $1.42 \times 10^{-3}$ \\
\hline 63.0 & $8.36 \times 10^{-3}$ & $8.20 \times 10^{-3}$ & $4.11 \times 10^{-4}$ & $3.22 \times 10^{-4}$ & $3.61 \times 10^{-3}$ & $6.42 \times 10^{-4}$ & $2.40 \times 10^{-3}$ \\
\hline 123.4 & $6.84 \times 10^{-3}$ & $6.38 \times 10^{-3}$ & $3.05 \times 10^{-4}$ & $4.43 \times 10^{-4}$ & $4.01 \times 10^{-3}$ & $6.30 \times 10^{-4}$ & $1.73 \times 10^{-3}$ \\
\hline 161.1 & $7.85 \times 10^{-3}$ & $6.09 \times 10^{-3}$ & $3.34 \times 10^{-4}$ & $5.23 \times 10^{-4}$ & $3.88 \times 10^{-3}$ & $7.49 \times 10^{-4}$ & $1.50 \times 10^{-3}$ \\
\hline 203.9 & 0.015 & $6.11 \times 10^{-3}$ & $2.69 \times 10^{-4}$ & $5.85 \times 10^{-4}$ & $3.51 \times 10^{-3}$ & $7.35 \times 10^{-4}$ & $1.74 \times 10^{-3}$ \\
\hline 251.8 & 0.017 & $5.85 \times 10^{-3}$ & $2.93 \times 10^{-4}$ & $4.83 \times 10^{-4}$ & $3.32 \times 10^{-3}$ & $6.24 \times 10^{-4}$ & $2.35 \times 10^{-3}$ \\
\hline 566.5 & 0.022 & 0.020 & $3.31 \times 10^{-4}$ & $4.24 \times 10^{-4}$ & $2.32 \times 10^{-3}$ & $6.86 \times 10^{-4}$ & $5.40 \times 10^{-3}$ \\
\hline 1000 & 0.037 & 0.027 & $1.12 \times 10^{-3}$ & $7.62 \times 10^{-4}$ & $1.46 \times 10^{-3}$ & $1.06 \times 10^{-3}$ & $5.66 \times 10^{-3}$ \\
\hline 2260 & 0.015 & 0.047 & $1.35 \times 10^{-3}$ & $2.51 \times 10^{-3}$ & $1.21 \times 10^{-3}$ & $1.17 \times 10^{-3}$ & $4.05 \times 10^{-3}$ \\
\hline 4000 & 0.012 & 0.039 & $1.12 \times 10^{-3}$ & $3.64 \times 10^{-3}$ & $1.83 \times 10^{-3}$ & $9.60 \times 10^{-4}$ & $2.73 \times 10^{-3}$ \\
\hline 6290 & 0.017 & 0.106 & $1.45 \times 10^{-3}$ & $3.78 \times 10^{-3}$ & $2.13 \times 10^{-3}$ & $7.47 \times 10^{-4}$ & $1.92 \times 10^{-3}$ \\
\hline 9060 & 0.029 & 0.165 & $1.93 \times 10^{-3}$ & $3.48 \times 10^{-3}$ & $2.09 \times 10^{-3}$ & $5.86 \times 10^{-4}$ & $1.43 \times 10^{-3}$ \\
\hline
\end{tabular}

Table S4 Charge transfer cross sections with regard to impact energy in proton-induced collisions with imidazole and AMI@ $\left(\mathrm{H}_{2} \mathrm{O}\right)$ n water clusters (in 10-16 $\left.\mathrm{cm}^{2}\right)$.

\begin{tabular}{|l|l|l|l|l|l|l|l|}
\hline $\mathrm{E}_{\text {lab }}(\mathrm{eV})$ & $\begin{array}{l}\text { 2-amino } \\
\text { imidazole }\end{array}$ & AMI@1W & AMI@2W & AMI@3W & AMI@4W & AMI@5W & AMI@6W \\
\hline 2.5 & $4.02 \times 10^{-4}$ & $1.57 \times 10^{-3}$ & 0.308 & 0.086 & $2.07 \times 10^{-3}$ & $1.37 \times 10^{-3}$ & $8.06 \times 10^{-4}$ \\
\hline 5.7 & $8.80 \times 10^{-4}$ & $1.55 \times 10^{-3}$ & 0.477 & 0.105 & $2.00 \times 10^{-3}$ & $2.30 \times 10^{-3}$ & $1.60 \times 10^{-3}$ \\
\hline 10.0 & $1.46 \times 10^{-3}$ & $1.38 \times 10^{-3}$ & 0.606 & 0.102 & $1.89 \times 10^{-3}$ & $2.57 \times 10^{-3}$ & $1.91 \times 10^{-3}$ \\
\hline
\end{tabular}




\begin{tabular}{|l|l|l|l|l|l|l|l|}
\hline 22.6 & $3.13 \times 10^{-3}$ & $1.34 \times 10^{-3}$ & 0.731 & 0.119 & $1.90 \times 10^{-3}$ & $3.32 \times 10^{-3}$ & $2.60 \times 10^{-3}$ \\
\hline 40.0 & $8.74 \times 10^{-3}$ & $1.36 \times 10^{-3}$ & 0.638 & 0.122 & $2.46 \times 10^{-3}$ & $5.84 \times 10^{-3}$ & $5.42 \times 10^{-3}$ \\
\hline 63.0 & $2.19 \times 10^{-2}$ & $1.06 \times 10^{-3}$ & 0.566 & 0.146 & $2.98 \times 10^{-3}$ & $7.43 \times 10^{-3}$ & $3.29 \times 10^{-3}$ \\
\hline 123.4 & $1.65 \times 10^{-2}$ & $1.23 \times 10^{-3}$ & 0.879 & 0.160 & $2.41 \times 10^{-3}$ & $6.72 \times 10^{-3}$ & $2.71 \times 10^{-3}$ \\
\hline 161.1 & $1.76 \times 10^{-2}$ & $1.37 \times 10^{-3}$ & 0.947 & 0.155 & $1.90 \times 10^{-3}$ & $6.09 \times 10^{-3}$ & $2.43 \times 10^{-3}$ \\
\hline 203.9 & $2.21 \times 10^{-2}$ & $1.40 \times 10^{-3}$ & 0.944 & 0.161 & $2.48 \times 10^{-3}$ & $5.50 \times 10^{-3}$ & $2.95 \times 10^{-3}$ \\
\hline 251.8 & $2.21 \times 10^{-2}$ & $1.32 \times 10^{-3}$ & 0.913 & 0.155 & $2.53 \times 10^{-3}$ & $5.10 \times 10^{-3}$ & $3.20 \times 10^{-3}$ \\
\hline 566.5 & $1.88 \times 10^{-2}$ & $1.04 \times 10^{-3}$ & 0.632 & 0.137 & $1.59 \times 10^{-3}$ & $6.56 \times 10^{-3}$ & $7.26 \times 10^{-3}$ \\
\hline 1000 & $1.80 \times 10^{-2}$ & $1.23 \times 10^{-3}$ & 0.406 & 0.091 & $1.16 \times 10^{-3}$ & $8.55 \times 10^{-3}$ & $1.28 \times 10^{-2}$ \\
\hline 2260 & $2.09 \times 10^{-2}$ & $1.79 \times 10^{-3}$ & 0.210 & 0.065 & $2.31 \times 10^{-3}$ & $7.99 \times 10^{-3}$ & $1.44 \times 10^{-2}$ \\
\hline 4000 & $6.20 \times 10^{-2}$ & $1.74 \times 10^{-3}$ & 0.142 & 0.110 & $2.76 \times 10^{-3}$ & $6.09 \times 10^{-3}$ & $1.22 \times 10^{-2}$ \\
\hline 6290 & $8.86 \times 10^{-2}$ & $1.48 \times 10^{-3}$ & 0.608 & 0.185 & $2.49 \times 10^{-3}$ & $4.87 \times 10^{-3}$ & $1.05 \times 10^{-2}$ \\
\hline 9060 & 0.110 & $1.23 \times 10^{-2}$ & 0.586 & 0.227 & $2.10 \times 10^{-3}$ & $4.06 \times 10^{-3}$ & $8.90 \times 10^{-3}$ \\
\hline
\end{tabular}

